\title{
Resistance Trends among Pseudomonas aeruginosa Isolates in a Tertiary Care Centre in South Gujarat
}

\author{
Nishi Tiwari*, Sangita Rajdev, Summaiya Mullan \\ Department of Microbiology, Government Medical College, Surat, India \\ Email: ^tiwarinishi23@gmail.com
}

How to cite this paper: Tiwari, N., Rajdev, S. and Mullan, S. (2017) Resistance Trends among Pseudomonas aeruginosa Isolates in a Tertiary Care Centre in South Gujarat. $A d$ vances in Microbiology, 7, 188-194. https://doi.org/10.4236/aim.2017.73015

Received: January 24, 2017

Accepted: March 6, 2017

Published: March 9, 2017

Copyright $\odot 2017$ by authors and Scientific Research Publishing Inc. This work is licensed under the Creative Commons Attribution International License (CC BY 4.0).

http://creativecommons.org/licenses/by/4.0/ (c) (i) Open Access

\begin{abstract}
It is necessary to determine the susceptibility pattern of clinical isolates especially nosocomial one in the clinical settings for making strategy for effective empirical treatment $\&$ to reduce incidence of multidrug resistant bugs. Aim of this study was to detect the antimicrobial susceptibility pattern of $P$. aeruginosa isolates from clinical samples between January 2014 to December 2015, received at department of Microbiology, GMC, Surat. Clinical isolates were confirmed as $P$. aeruginosa by phenotypic methods/Vitek2 compact system as per availability. Genetic sequencing could not be performed due to unavailability. Antimicrobial susceptibility tests were performed by Kirby-Bauer disc diffusion method/Vitek2 compact system \& Interpretation was done according Clinical and Laboratory Standards Institute (CLSI) of that year [1] [2]. Seven hundred fifty seven $P$. aeruginosa strains were studied during the study period. Most of the isolates were from surgery ward (62\%), followed by orthopaedic ward (15\%). $65 \%$ of the total isolates were from swab samples followed by urine (7\%), pus, fluid (5\%) \& devices (4\%). $60 \%$ isolates were resistant to Ceftazidime \& for other drugs resistance pattern was as follows: Cefepime (52\%), Levofloxacin (49\%), Ticarcillin/clavulanic acid (49\%), Meropenem \& Gentamycin (44\%), Ciprofloxacin (43\%), Amikacin (41\%), Tobramycin (39\%), Netlimycin (36\%), Piperacillin (32\%), Aztreonam (31\%), Piperacillin/tazobactam (26\%), Imipenem (23\%), Doripenem (12\%) \& Gatifloxacin $(10 \%)$. As there is predominance of isolates from surgical ward in present study \& resistance to carbapenem group of drugs was also found, indicating that most of the infection caused by Pseudomonas aeruginosa may be nosocomial.
\end{abstract}

\section{Keywords}

$P$. aeruginosa, Antimicrobial Sensitivity, Drug Resistance 


\section{Introduction}

There is increase in multidrug resistance in clinical isolates in all the clinical settings these days. It is matter of concern as it can become a cause of threat to public health. There is need for making strategies that are efficient to prevent further increase of drug resistance in clinical isolates. Regular monitoring of antimicrobial susceptibility pattern of clinical isolates can greatly contribute in making preventive strategies for multidrug resistance. Prevalence of $P$. aeruginosa is $15 \%-80 \%$ in chronic wounds [3]. P. aeruginosa is an organism that is present in many diverse environmental settings. It has ability to survive on minimal nutritional requirements and it can tolerate a variety of physical conditions. All these properties have allowed this organism to persist in both community and hospital settings. $P$. aeruginosa can be isolated from a variety of sources in hospital settings like respiratory therapy equipment, antiseptics, soap, sinks, mops, medicines, and physiotherapy and hydrotherapy pools [4]. Pseudomonas aeruginosa is an important cause of hospital acquired infection due to all these properties. $P$. aeruginosa \& $P$. maltophila are causative agents of $80 \%$ opportunistic infections caused by Pseudomonas [5]. It causes wide variety of infections. There is availability of various antimicrobial agents with anti-pseudomonas activity these days but Peudomonas aeruginosa is still an important cause of morbidity \& mortality in hospitalized patients. Therefore, this study has been carried out to study the current antimicrobial sensitivity pattern of Pseudomonas aeruginosa in patients attending New Civil Hospital, Surat.

\section{Material \& Method}

It was a retrospective study done in Department of Microbiology, GMC, Surat. Antibiotic susceptibility pattern of $P$. aeruginosa isolated from the clinical samples of patients attending the New Civil Hospital, those were received in department of Microbiology, GMC, Surat during period of January 2014-December 2015 were analyzed. Repeat isolates from same sample of patient within 2 days of isolation of first isolate were not included in study (there is no standard currently available which tell about optimal frequency to do repeat antimicrobial susceptibility testing of repeat isolate from same sample when an organism is isolated from the same specimen source repeatedly but, testing every 2 to 5 days is thought sufficient) [6]. Species level identification was done by phenotypic methods (oxidase test, growth at $42^{\circ} \mathrm{C}$, TSI test, arginine decarboxylation, OF sugar fermentation test) \& Vitek 2 compact system as per availability. Genetic sequencing could not be done due to unavailability. Antimicrobial susceptibility was done by Kirbybauer technique (Figure 1)/Vitek 2 compact systemas per availability. Antimicrobial tested are Pipercillin (100 ug), Pipercillin/Tazobactam (100/10ug), Ticarcillin/Clavulinic acid (75/10ug), Ceftazidime (30 ug), Cefipime (30 ug), Aztreonam (30 ug), Doripenem (10 ug), Imipenem (10 ug), Meropenem (10 ug), Gentamycin (10 ug), Tobramycin (10 ug), Amikacin (30 ug), Netilimycin (30 ug), Ciprofloxacin (5 ug), Levofloxacin (5 ug) \& Gatifloxacin (5 ug) according to CLSI guideline 2014 \& 2015 [1] [2]. For Kirbybauer technique Mul- 

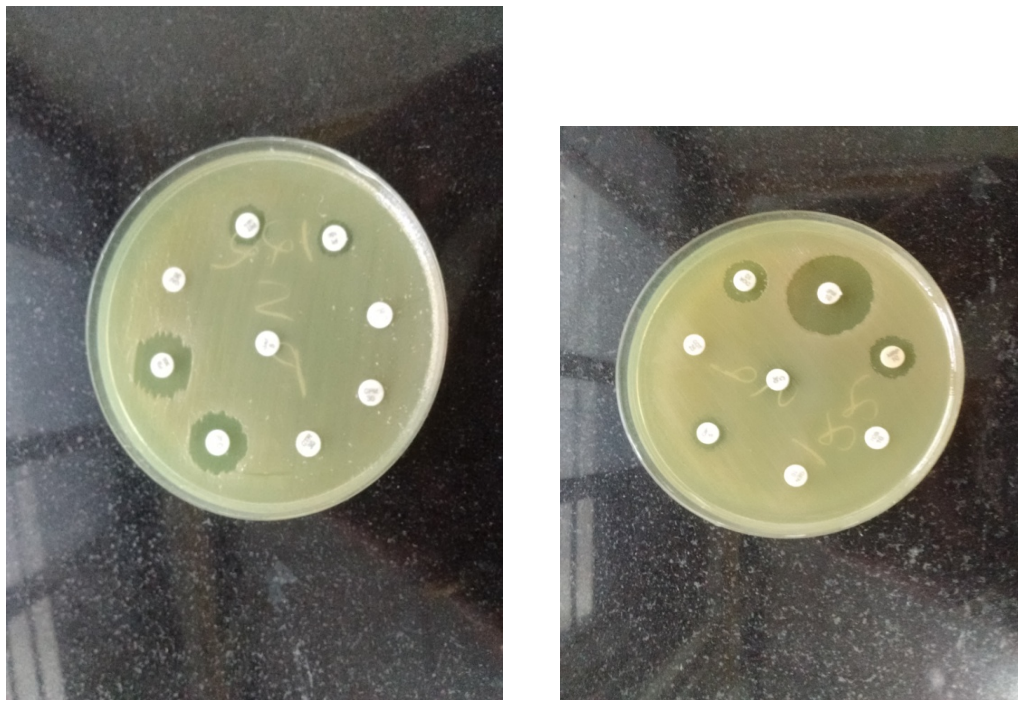

Figure 1. Showing antimicrobial susceptibility testing of $P$. aeruginosa by Kirby-bauer technique.

ler Hinton agar( HiMedia) medium having $\mathrm{pH} 7.2$ to 7.4 at room temperature was used. Before doing antimicrobial susceptibility testing it was dried to remove droplet of moisture but taking care of not let it overdry. The antibiotic disks were maintained at $-20^{\circ} \mathrm{C}$ until needed, according to the manufacturer's recommendations. Antibiotic disks were allowed to come at room temperature before use. They were checked for expiry date \& quality control testing was done time to time for ensuring correct results. Antibiotic susceptibility testing was done as follows:

4 to 5 well-isolated colonies of the same morphological type from an agar plate were selected from 18 - 24 hour old culture. Top of each colony was touched with a with a wire loop and transferred to a tube containing 4 to $5 \mathrm{~mL}$ of peptone water. This suspension was incubated at $37^{\circ} \mathrm{C}$ for 2 hours. Turbidity of suspension was adjusted to $0.5 \mathrm{McF}$ arland standard. A sterile non toxic swab was dipped into this suspension \& then rotated \& pressed several times on inside wall of test tube above the level of fluid to remove excess inoculums. Dried surface of Muller Hinton agar was inoculated with the swab three times at $60^{\circ}$ from previous stroke. Plate was allowed to dry for about 5 minutes to remove the excess moisture. Antibiotic discs were placed appropriately on agar surface (minimum 24 $\mathrm{mm}$ distance between discs \& $15 \mathrm{~mm}$ distance between disc \& margin of plates). On one $120 \mathrm{~mm}$ plate 6 antibiotic discs were placed. Once the disc was placed care was taken not to move the disk. Then plate was put in incubator at $37^{\circ}$ in inverted position. Incubation was done for 16-18 hours aerobically. Zone of inhibition was measured to the nearest millimeter using scale. Results were interpreted according to CLSI guidelines 2015 \& 2016 respectively. Results were entered in Excel sheet \& analysis was performed.

\section{Result}

Pseudomonas aeruginosa comprised of $23 \%$ (757 out of 3329) of all gram nega- 
tive organism isolated from various clinical samples received in department of microbiology, GMC, Surat from January 2014-December 2015. Ward-wise analysis showed that most of the isolates were from surgery ward (62\%), followed by orthopaedic ward (15\%) (Figure 2). Sample-wise analysis showed that $65 \%$ of the total isolates were from swab samples followed by urine (7\%), pus \& fluid (5\%), \& devices (4\%) (Figure 3). Most of the isolates were resistant to Ceftazidime $(60 \%) \&$ for other drugs resistance pattern was as follows: Cefepime (52\%), Levofloxacin (49\%), Ticarcillin/clavulanic acid (49\%), Meropenem \& Gentamycin (44\%), Ciprofloxacin (43\%), Amikacin (41\%), Tobramycin (39\%), Netlimycin (36\%), Piperacillin (32\%), Aztreonam (31\%), Piperacillin/tazobactam (26\%), Imipenem (23\%), Doripenem (12\%) \& Gatifloxacin (10\%) (Figure 4).

\section{Discussion}

Prevalence rate of Pseudomonas aeruginosa was $23 \%$ (757 out of 3329) of all gram negative organism isolated from various clinical samples received in department of microbiology, GMC, Surat from January 2014-December 2015. Most of the isolates were from Surgery ward (62\%), followed by Orthopaedic ward (15\%). Sample-wise analysis showed that $65 \%$ of the total isolates were from swab samples followed by urine (7\%), pus \& fluid (5\%), \& devices (4\%). These findings are similar with Muktikesh Das et al. who showed 67.6\% isolation rate of $P$. aeruginosa from pus \& swab samples. In present study isolation rate

\section{Ward-wise isolation rate of P.aeruginosa}

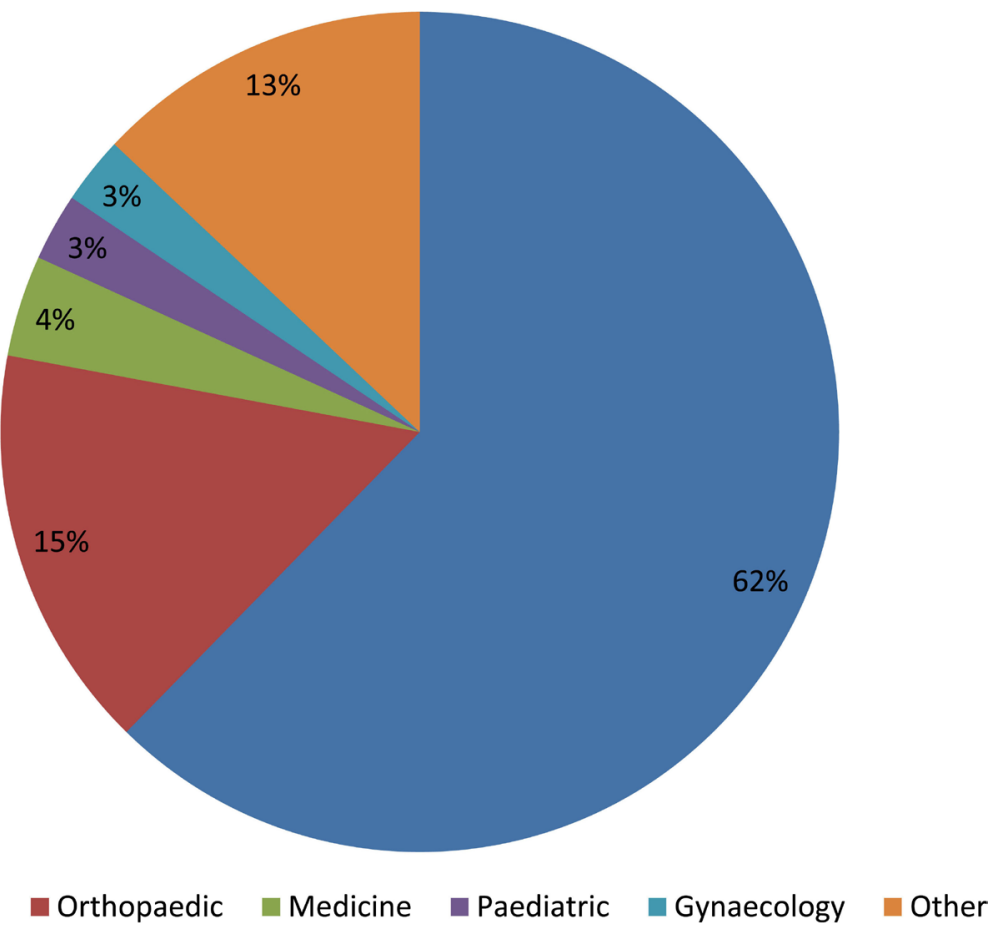

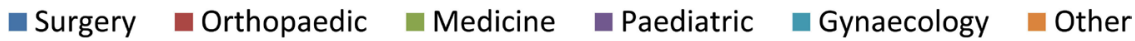

Figure 2. Ward-wise isolation rate of P.aeruginosa in present study. Showing ward-wise isolation rate of $P$. aeruginosa. Most of the isolates were from surgery ward (62\%), followed by orthopaedic ward (15\%), medicine (4\%), Paediatric (3\%), Gyanecology (3\%) \& other (13\%). 
Sample-wise isolation rate of $P$.aeruginosa

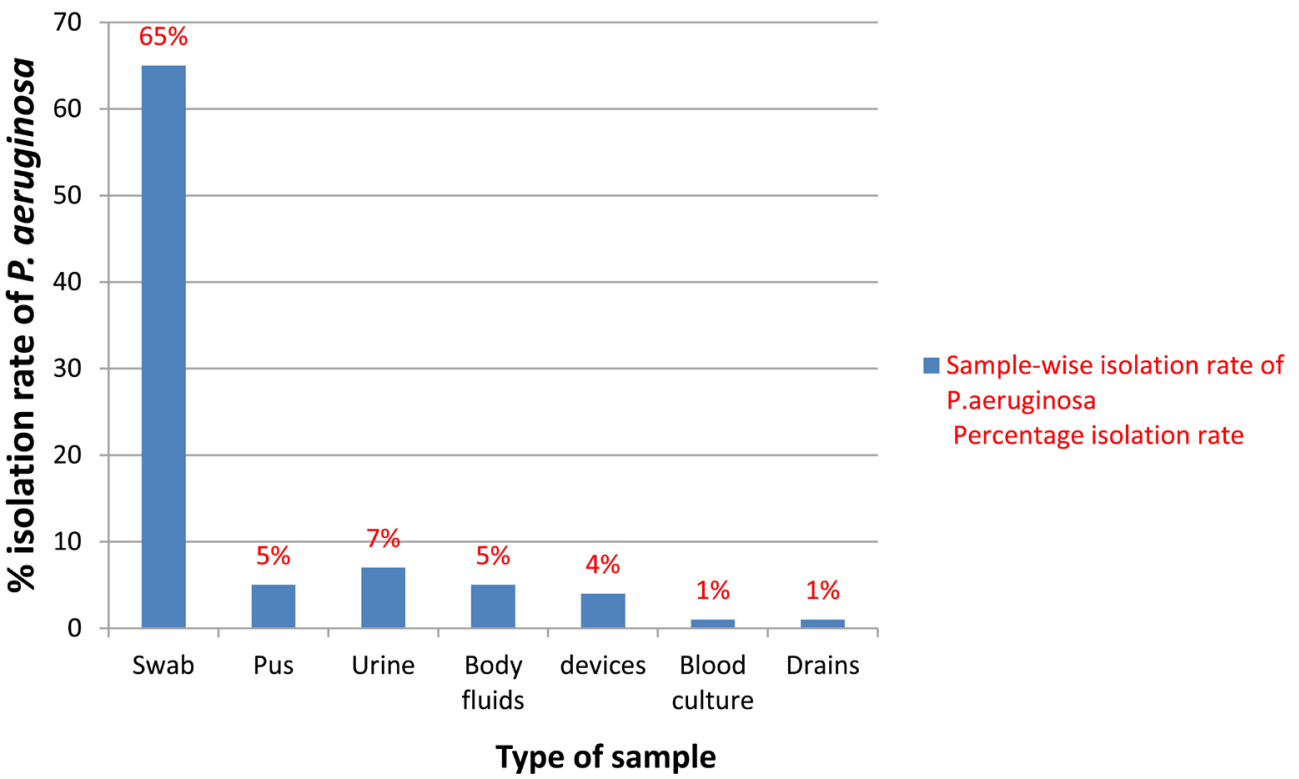

Figure 3. Sample-wise isolation rate of $P$. aeruginosa in present study. (Showing isolation rate of $P$. aeruginosa from various clinical samples in present study. Type of sample is plotted on $\mathrm{X}$-axis \& percentage isolation rate is plotted on $\mathrm{Y}$-axis. Highest rate of isolation was from samples followed by urine (7\%), pus \& fluid (5\%), \& devices (4\%), Blood culture \& drains (1\%).).

\section{Antimicrobial resistance pattern of P.aeruginosa isolates}

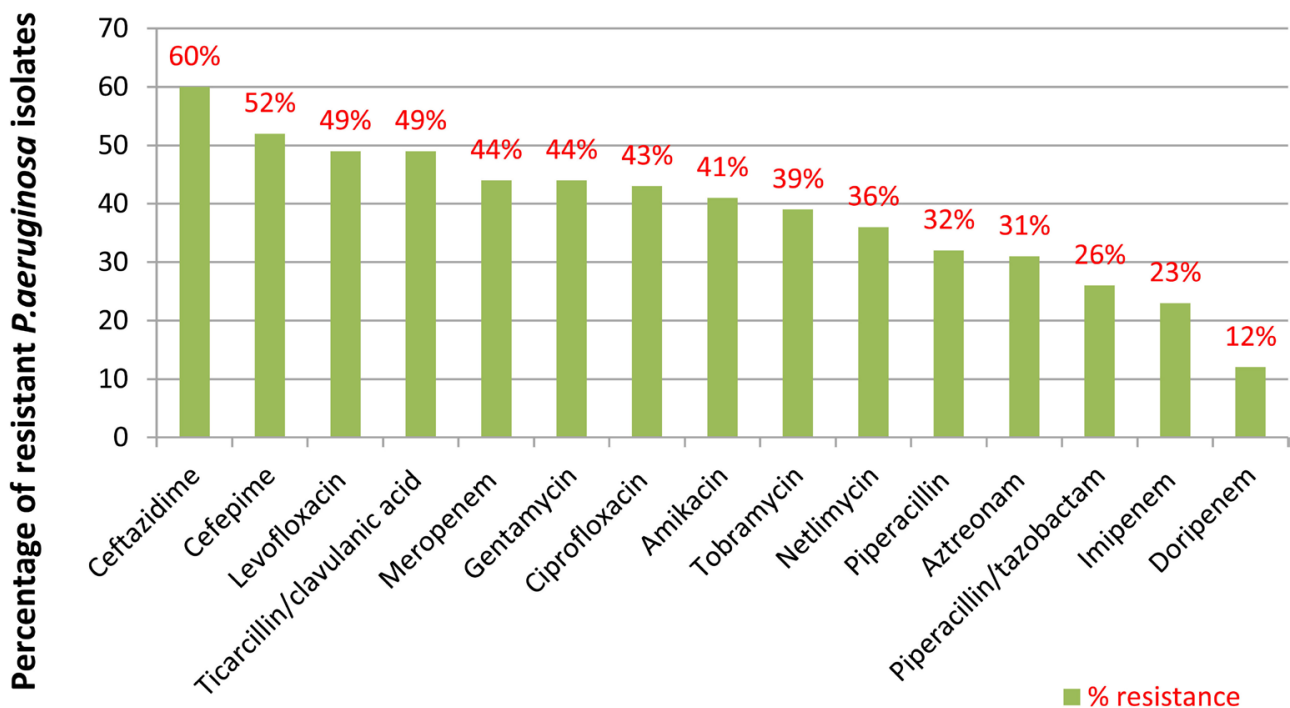

Antimicrobials used for analysis of resistance pattern of P.aeruginosa

Figure 4. Antimicrobial resistance pattern of $P$. aeruginosa isolates in present study. (Showing antimicrobial resistance pattern of $P$. aeruginosa isolates in present study. On X-axis antimicrobial tested in present study are plotted. On Y-axis percentage of resistant $P$. aeruginosa isolates to antimicrobials is plotted. $60 \%$ of the isolates were resistant to Ceftazidime \& for other drugs resistance pattern was as follows: Cefepime (52\%), Levofloxacin (49\%), Ticarcillin/clavulanic acid (49\%), Meropenem \& Gentamycin (44\%), Ciprofloxacin (43\%), Amikacin (41\%), Tobramycin (39\%), Netlimycin (36\%), Piperacillin (32\%), Aztreonam (31\%), Piperacillin/tazobactam (26\%), Imipenem (23\%), Doripenem (12\%) \& Gatifloxacin (10\%).) 
of $P$. aeruginosa from urine sample was 7\%, which is less than previous studies carried out by Sheetal Sharma et al. (2016), Amandeep kaur et al. (27.1\%), Sunal Lakum et al. (20\%), Muktikesh Das et al. (15\%) [7] [8] [9] [10]. These studies also showed high isolation rate of $P$. aeruginosa from pus sample. Most of the isolates in present study were resistant to Ceftazidime (60\%). O. A. Igbalajobi et al. (73.8\%), Amandeep Kaur et al. (62.8\%), Sonal Lakum et al. (45.95\%), Muktikesh Das et al. (77.7\%) \& Javed R. Al Zaidi et al. (100\%) have shown similar high degree resistance of $P$. aeruginosa to ceftazidime [8] [9] [10] [11] [12]. For other drugs resistance pattern was as follows: Cefepime (52\%), Levofloxacin (49\%), Ticarcillin/clavulanic acid (49\%), Meropenem \& Gentamycin (44\%), Ciprofloxacin (43\%), Amikacin (41\%), Tobramycin (39\%), Netlimycin (36\%), Piperacillin (32\%), Aztreonam (31\%), Piperacillin/tazobactam (26\%), Imipenem (23\%), Doripenem (12\%) \& Gatifloxacin (10\%). Resistance to carbapenems was also present in this study \& it was ranges from $12 \%-44 \%$ for different drugs of this group. This high degree of resistance of $P$. aeruginosa for carbapenem group of drugs was also found in previous studies. Nandkuduru Premanandham et al. reported 55.56\% resistance to doripenem \& $25.8 \%$ for Imipenem, Amandeep Kaur et al. reported 33\% resistance to meropenem \& $17.8 \%$ resistance to Imipenem, Oman Basir Ahmed et al. reported 25\% resistance to Imipenem \& Sonal Lakum et al. reported $21.6 \%$ resistance to Imipenem for $P$. aeruginosa isolates [8] [9] [13] [14]. This increasing drug resistance to $P$. aeruginosa isolates is a matter of great concern for medical fraternity. As most of the isolates in present study were from surgical ward, hospital acquired or iatrogenic cause seems to play a main role. Increasing carbapenem resistance is also seen that arise a need to take further steps to do antibiotic susceptibility testing before giving empirical therapy $\&$ prevent abuse of antimicrobial therapy.

\section{Limitations}

1) Modified hodge test could not be done to confirm carbapenemase resistance.

2) There is need to do molecular study to identify resistant gene prevalent in setting.

\section{Conclusion}

Injudicious use of drugs is one of main contributors to emerging resistance of $P$. aeruginosa to antimicrobial therapy. It also contributes to appearance of multidrug resistant strains of bacterium. Most of the isolates in present study were from surgical ward \& isolated mainly in swab sample. Resistance to carbapenem group of drugs was also found. These findings indicate that most of the infection caused by Pseudomonas aeruginosa may be iatrogenic or hospital acquired.

\section{References}

[1] Clinical and Laboratory Standards Institute (2014) Performance Standards for Antimicrobial Susceptibility Testing; 21th Informational Supplement (M100-S24). Clinical and Laboratory Standards Institute, Wayne.

[2] Clinical and Laboratory Standards Institute (2015) Performance Standards for An- 
timicrobial Susceptibility Testing; 21th Informational Supplement (M100-S25). Clinical and Laboratory Standards Institute, Wayne.

[3] Korgaonkar, A., Trivedi, U., Rumbaugh, K.P. and Whiteley, M. (2013) Community Surveillance Enhances Pseudomonas aeruginosa Virulence during Polymicrobial Infection. Proceedings of the National Academy of Sciences of the United States of America, 110, 1059-1064. https://doi.org/10.1073/pnas.1214550110

[4] Raj, M.L., Kalaigandhi, V. and Kanagaraj, C. (2015) The Occurrence of (MDR/ MDS) Pseudomonas aeruginosa among Nosocomial and Community Acquired Infections in and around Coimbatore, India. International Journal of Current Microbiology and Applied Sciences, 4, 753-761.

[5] Iglewski, B.H. (1996) Pseudomonas. In: Baron, S., Ed., Medical Microbiology, 4th Edition, Chapter 27, University of Texas Medical Branch at Galveston, Galveston.

https://www.ncbi.nlm.nih.gov/books/NBK8326/

[6] Giltner, C.L., Kelesidis, T., Hindler, J.A., Bobenchik, A.M. and Humphries, R.M. (2014) Frequency of Susceptibility Testing for Patients with Persistent MethicillinResistant Staphylococcus aureus Bacteremia. Journal of Clinical Microbiology, 52, 357-361. https://doi.org/10.1128/JCM.02081-13

[7] Sharma, S. and Srivastava, P. (2016) Resistance of Antimicrobial in Pseudomonas aeruginos. International Journal of Current Microbiology and Applied Sciences, 5, 121-128. https://doi.org/10.20546/ijcmas.2016.503.017

[8] Kaur, A., Singh, S., Gill, A.K. and Kaur, N.(2016) Prevelance \& Antimicrobial Susceptibility Pattern of Pseudomonas aeruginosa Isolated from Various Clinical Samples in Tertiary Care Hospital, Bathinda. Indian Journal of Basic and Applied Medical Research, 5, 777-784.

[9] Lakum, S.A., Pandya, H., Shah, K. and Lakhani, S.J. (2016) Antibiotic Susceptibility Pattern of Pseudomonas aeruginosa at the Tertiary Care Center, Dhiraj Hospital, Piparia, Gujarat. International Archives of Integrated Medicine, 3, 133-137.

[10] Dash, M., Padhi, S., Narasimham, M.V. and Pattnaik, S. (2014) Antimicrobial Resistance Pattern of Pseudomonas aeruginosa Isolated from Various Clinical Samples in a Tertiary Care Hospital, South Odisha, India. Saudi Journal for Health Sciences, 3, 15-19. https://doi.org/10.4103/2278-0521.130200

[11] Igbalajobi, O.A., Oluyege, A.O., Oladeji, A.C. and Babalola, J.A. (2016) Antibiotic Resistance Pattern of Pseudomonas aeruginosa Isolated from Clinical Samples in Ekiti State University Teaching Hospital, Ado-Ekiti, Ekiti State of Nigeria. British Microbiology Research Journal, 12, 1-6. https://doi.org/10.9734/BMRJ/2016/22515

[12] Al-Zaidi, J.R. (2016) Antibiotic Susceptibility Patterns of Pseudomonas aeruginosa Isolated from Clinical and Hospital Environmental Samples in Nasiriyah, Iraq. African Journal of Microbiology Research, 10, 844-849.

https://doi.org/10.5897/AJMR2016.8042

[13] Premanadham, N., Jitendra, K., Reddi, M.S.P. and Kumar, C. (2016) Antibiotic Resistance Pattern of Pseudomonas aeruginosa Strains Isolated from Blood CulturesBatec/Alert 3D in a Tertiary Care Centre Narayana Hospital \& Medical College Nellore AP, India. International Journal of Current Microbiology and Applied Sciences, 5, 263-268. https://doi.org/10.20546/ijcmas.2016.511.028

[14] Ahmed, O.B. (2016) Incidence and Antibiotic Susceptibility Pattern of Pseudomonas aeruginosa Isolated from Inpatients in Two Tertiary Hospitals. Clinical Microbiology, 5, 248. 
Submit or recommend next manuscript to SCIRP and we will provide best service for you:

Accepting pre-submission inquiries through Email, Facebook, LinkedIn, Twitter, etc. A wide selection of journals (inclusive of 9 subjects, more than 200 journals)

Providing 24-hour high-quality service

User-friendly online submission system

Fair and swift peer-review system

Efficient typesetting and proofreading procedure

Display of the result of downloads and visits, as well as the number of cited articles Maximum dissemination of your research work

Submit your manuscript at: http://papersubmission.scirp.org/

Or contact aim@scirp.org 